\title{
Complejidad textual con fines académicos en el ámbito de la traducción. Variables para su clasificación
}

\section{(Textual complexity when translating English to Spanish texts for academic purposes. Variables to their classification)}

\author{
FERNANDO MUÑOZ LÓPEZ \\ fmunozl@inacap.cl \\ IN ACAP
}

Fecha de recepción: 26 de abril de 2019

Fecha de aceptación: 21 de octubre de 2019

Resumen: La actividad traductora es de por sí un proceso complejo en cuanto al conocimiento. Para lograr llevar el mensaje de una lengua a otra, existen convenciones lingüísticas y culturales que deben ser consideradas. La complejidad en el ámbito terminológico (Cabré 2003) es una variable que puede incidir directamente en el aprendizaje tanto del proceso traductor como del aprendizaje de una lengua, si no existen criterios pedagógicos claros sobre la selección de material académico para el educando. El objetivo de esta investigación fue crear un instrumento académico para la categorización y clasificación de niveles de complejidad de textos considerando los criterios baja, media y alta complejidad, y así intentar estandarizar el proceso de selección de material para aplicar metodologías a programas universitarios de la carrera de traducción. Con lo anterior, se busca definir criterios elementales, en formato de material instruccional, para que en cada programa de estudio donde se vea reflejada la competencia del saber-hacer ligada a la traducción, la selección de textos de la lengua meta no dependa de la intuición personal o conocimiento experto, sino de características fundamentales de selección académica para poder cumplir con las competencias y aprendizajes esperados de los programas de estudio de la carrera de traducción en la INACAP Chile y su perfil de egreso.

Palabras clave: Traducción. Complejidad terminológica. Nivel de especialidad. Texto de lengua meta. Criterios.

Abstract: Translation activity is by nature a complex process in terms of knowledge. There are linguistic rules and cultural guidelines in order to 
communicate one's message to another language. Terminology complexity (Cabré 2003) is an important variable that can directly affect translation learning and second language acquisition, when not considering teaching criteria in selecting academic texts for the students. The final objective of this project was to create an instrument that allows teachers to select and classify the complexity levels stated in each translation class program for low, intermediate and high complexity texts. This is to standardize the text selection process to which the students are exposed to when studying translation at INACAP University. The project aims to define the fundamental criteria in an instructional portfolio, so as each program can develop a competence-based approach related to the translation, the selection of source texts to translate is not up to each teacher's expert opinion, but to the fundamental features of the academic text selection. All this is in order to accomplish the objective of each class program and achieve the exit profile.

Keywords: Translation. Terminology complexity. Level of specialization. Source language texts. Criteria.

\section{Complejidad y traducción}

La adquisición de la competencia traductora (Mayoral 2005: 165) se entiende como el proceso de traducir de forma efectiva de una lengua a otra, considerando que las competencias se encuentran en constante estado de desarrollo y perfeccionamiento. Generalmente la adquisición de habilidades asociadas a la competencia traductora está ligada al nivel de adquisición de una segunda lengua, y es donde se hace inherente el cruce entre el lenguaje y el conocimiento cultural. Dicha adquisición será parte fundamental del desarrollo de la actividad traductora, especialmente en estudiantes nativos del castellano cuando aprenden una lengua como el inglés. El Common European Framework of Reference destaca dicha adquisición como un elemento inclusivo en sus distintos descriptores por niveles, en los cuales se destaca el aprendizaje orientado a la acción, la adaptabilidad del estudiante para un aprendizaje continuo, para inserarse en un contexto social de forma adecuada (CEFR 2018). Entendiendo entonces que la tarea traductora forma parte de un circuito de aprendizajes multidisciplinares, es dicha característica la que la convierte en una habilidad específica, con desarrollo de competencias particulares que requieren de una guía determinada por nivel de especialización específico.

El lenguaje especializado requiere desarrollo de habilidades documentales e instrumentales individuales para perfiles profesionales; el conocimiento especializado permite la adquisición del concepto de estar "familiarizado", ya que 
Complejidad textual con fines académicos en el ámbito de la traducción...

la funcionalidad del lenguaje se va a ver limitada solamente por contextos especializados en los que se suscribe. Dentro del lenguaje especializado consideraremos el uso de una segunda lengua como parte de un grado de especialización. En el momento de contextualizar el uso del inglés como segunda lengua para propósitos específicos, se indica que adoptar etiquetas o normas para caracterizar oraciones inglesas en español siguiendo una estructura similar es equívoco (Yule 2007). Las categorías, reglas y normas que posee el inglés difieren en tratamiento para su aprendizaje y producción. En este artículo se considera como vínculo inherente la importancia de la competencia documental en el desarrollo de traductores en instituciones académicas según García-Yebra (2004). En su trabajo, plantea "la necesidad de incluir esta disciplina científica en los planes de estudio de la licenciatura vino marcada en su momento por un amplio abanico de razones teórico-practicas, que demostraron que la documentación se convierte en un elemento clave del proceso traslativo" (García 2004: 275).

Considerando la fase heurística de la investigación, entendemos que no existe un nivel de estandarización sobre los grados de complejidad tanto terminológica como textual para la selección de textos en programas académicos de pregrado, y tampoco se encuentran establecidos en distintas partes de un currículum en programas de traducción. Si se consideran conceptos como los de complejidad y densidad terminológica (Cabré 1992), estos resultarían fundamentales en la didáctica de la traducción en relación con la selección de criterios o campos del conocimiento, ya que la no consideración de los mismos podría elevar el juicio experto a la cumbre de las decisiones a la hora de establecer criterios de selección de material, siendo necesario un criterio objetivo para la selección textual para la enseñanza de la traducción como competencia. El juicio experto puede llevar a establecer estrategias para la realización de procedimientos específicos, pero se requiere de una evaluación teórica para poder sistematizar un modelo que cumpla con elementos específicos con el fin de poder concretizar una acción (Cabrero y Llorente 2013). Esta falta de saberes concretos, muchas veces en la enseñanza de traductores, podría llevar a entregar material de trabajo de traducción, que no se ajusta al nivel de comprensión que posee el aprendiz de traducción.

El grado de especialización y la densidad terminológica son conceptos clave en el desarrollo de competencias del terminólogo en el ámbito de la traducción, ya que las consideraciones subyacentes en los conceptos entregan las bases de entendimiento e importancia de las palabras y términos en un texto, fundamentales para una traducción natural y funcional. Para lograr el propósito original en una traducción, estas consideraciones pueden ser parte de un todo para determinar la finalidad última de un texto (Vermeer 2004). A continuación, se procede a operacionalizar el concepto de complejidad con fines académicos, 
para la efectiva selección de textos de acuerdo con el nivel de adquisición de una segunda lengua, para propósitos traductores, específicamente para la adquisición efectiva del proceder traductor con respecto a una lengua extranjera.

\section{Complejidad textual}

En primer lugar, se definieron parámetros para la construcción de un instrumento con el que se testearía la complejidad de textos para propósitos específicos. Como base se consideraron programas de estudio de carreras relacionadas con el nivel de inglés o el desarrollo de la carrera de traducción. Para esto, se tomaron indicadores académicos del Programa de Traducción de INACAP Chile (INACAP s.f.), donde existe un nivel de inglés asociado a cada semestre de estudio, en donde la adquisición de la competencia traductora va de la mano del desarrollo y escalamiento de niveles de inglés según el Marco Común Europeo de Referencia (CEFR). Por ello, se definirá un proceso de selección, porque se entiende que la adquisición de la competencia traductora va de la mano del conocimiento o dominio que tenga una estudiante con respecto a la lengua que está adquiriendo:

\begin{tabular}{|l|l|l|l|l|l|l|}
\hline $\begin{array}{l}2^{\circ} \\
\text { semestre }\end{array}$ & $\begin{array}{l}\text { 3er } \\
\text { semestre }\end{array}$ & $\begin{array}{l}4^{\circ} \\
\text { semestre }\end{array}$ & $\begin{array}{l}5^{\circ} \\
\text { semestre }\end{array}$ & $\begin{array}{l}6^{\circ} \\
\text { semestre }\end{array}$ & $\begin{array}{l}7^{\circ} \\
\text { semestre }\end{array}$ & $\begin{array}{l}8^{\circ} \\
\text { semestre }\end{array}$ \\
\hline $\begin{array}{l}\text { Taller de } \\
\text { traducci } \\
\text { ón I }\end{array}$ & $\begin{array}{l}\text { Taller de } \\
\text { traducci } \\
\text { ón II }\end{array}$ & $\begin{array}{l}\text { Taller de } \\
\text { traducci } \\
\text { ón III }\end{array}$ & $\begin{array}{l}\text { Taller de } \\
\text { traducci } \\
\text { ón IV }\end{array}$ & $\begin{array}{l}\text { Taller de } \\
\text { traducci } \\
\text { ón del } \\
\text { español } \\
\text { al inglés }\end{array}$ & $\begin{array}{l}\text { Taller de } \\
\text { traducción } \\
\text { en negocios } \\
\text { internacional } \\
\text { es }\end{array}$ & $\begin{array}{l}\text { Taller } \\
\text { integral } \\
\text { de } \\
\text { traducci } \\
\text { ón }\end{array}$ \\
\hline 72 horas & 72 horas & 72 horas & 72 horas & 72 horas & 72 horas & 72 horas \\
\hline Alte 1 & Alte 2 & Alte 2 & Alte 3 & Alte 3 & Alte 4 & Alte 4 \\
\hline
\end{tabular}

Para esta investigación, se consideraron los niveles de adquisición receptivos del lenguaje (Waring 1997) dadas las características del proceso traductor, en cuanto a la comprensión de textos para llevar a cabo el proceso en sí. Ya considerando todas las nociones del inglés para el proceso traductor, se procedió a establecer criterios de selección que articularan específicamente con el cruce de información de los programas de estudio, en particular de INACAP y los niveles establecidos por ALTE Framework (2002). Para lo anterior, se consideraron los conceptos de comprender, leer y rastrear para poder encontrar criterios que pudiesen articular con el objetivo del trabajo. Al realizar el cruce de información, se procedió a establecer los criterios y las dimensiones de establecimiento de criterios para clarificar la complejidad. Dichos criterios se enmarcan en consideraciones sobre terminología de Cabré (1992), y legibilidad ARI 
Complejidad textual con fines académicos en el ámbito de la traducción...

(Automated Readability Index 1967). Para la terminología, se procedió a desglosar elementos fundamentales de características textuales para ámbitos generales, ya que la selección de material basada en complejidad no debiese ser en extremo complejo para los académicos, sino contribuir a una mejor experiencia académica en preparación de materiales para estudiantes de traducción. La dimensión de terminología se define por densidad y nivel de especialización. Dichas dimensiones están vinculadas al proceso de selección de textos en sí, considerando que profesores que enseñan talleres de traducción posean un perfil relacionado con la especialidad. Esto resultará ser fundamental ya que, si el académico no cumple con un perfil de asignatura de especialidad, no se podrá alcanzar las competencias a desarrollar. Por tanto, considerando el perfil y la terminología, se procede a establecer lo siguiente:

a) Densidad terminológica: la cantidad de términos específicos identificados supera el umbral de 4 términos por cada 10 palabras.

La justificación de dicha dimensión y su definición se basó en lo anteriormente expuesto y considerando la siguiente tabla en Cabré (1992):

\begin{tabular}{|c|c|c|c|}
\hline & $\begin{array}{l}\text { Texto con alta } \\
\text { densidad } \\
\text { terminológica }\end{array}$ & $\begin{array}{l}\text { Texto con densidad } \\
\text { terminológica } \\
\text { media }\end{array}$ & $\begin{array}{l}\text { Texto con densidad } \\
\text { terminológica baja }\end{array}$ \\
\hline $\begin{array}{l}\text { Desde } 200 \text { a } \\
500 \text { palabras }\end{array}$ & $\begin{array}{l}40 \% \text { o más de } \\
\text { términos del total }\end{array}$ & $\begin{array}{l}\text { Entre } 20 \% \text { y } 29 \% \\
\text { de términos del } \\
\text { total }\end{array}$ & $\begin{array}{l}\text { Menos del } 19 \% \text { de } \\
\text { terminología del } \\
\text { total }\end{array}$ \\
\hline $\begin{array}{l}\text { Desde } 501 \text { a } \\
1000 \text { palabras }\end{array}$ & $\begin{array}{l}30 \% \text { o más de } \\
\text { términos del total }\end{array}$ & $\begin{array}{l}\text { Entre } 20 \% \text { y } 29 \% \\
\text { de términos del } \\
\text { total }\end{array}$ & $\begin{array}{l}\text { Menos del } 19 \% \text { de } \\
\text { terminología del } \\
\text { total }\end{array}$ \\
\hline $\begin{array}{l}\text { Desde } 1001 \text { a } \\
2000 \text { palabras }\end{array}$ & $\begin{array}{l}30 \% \text { o más de } \\
\text { términos del total }\end{array}$ & $\begin{array}{l}\text { Entre } 20 \% \text { y } 29 \% \\
\text { de términos del } \\
\text { total }\end{array}$ & $\begin{array}{l}\text { Menos del } 19 \% \text { de } \\
\text { terminología del } \\
\text { total }\end{array}$ \\
\hline $\begin{array}{ll}\text { Hasta } & 4000 \\
\text { palabras } & \end{array}$ & $\begin{array}{l}30 \% \text { o más de } \\
\text { términos del total }\end{array}$ & $\begin{array}{l}\text { Entre } 20 \% \text { y } 29 \% \\
\text { de términos del } \\
\text { total }\end{array}$ & $\begin{array}{l}\text { Menos del } 19 \% \text { de } \\
\text { terminología del } \\
\text { total }\end{array}$ \\
\hline
\end{tabular}

Cuadro 1: propuesta inicial de categorización de densidad terminológica

El elemento fundamental para dicha consideración es la cantidad de palabras del texto. En los programas de estudio se sugiere en términos de sugerencia metodológica que los textos para práctica y pruebas fluctúen entre 200 y 1000 palabras (lo último para proyectos de traducción), por lo que el investigador consideró la definición de la dimensión como 4 cada 10 términos basado en la complejidad textual que convergen en textos con alto grado de terminología y el 
uso de fuentes de información especializada. A continuación, se procedió con la justificación de la dimensión legibilidad según índices automáticos. Esta dimensión considera ya no la terminología, sino la cantidad de oraciones, palabras y la construcción del texto, otorgando ciertos niveles de dificultad según la composición sintáctica del texto. A mayor cantidad de oraciones, sílabas y mayor cantidad de oraciones de dichos elementos, mayor grado de dificultad para el lector. Para esto se tomaron las consideraciones de ARI (1967):

a) El contexto del lector es importante para determinar un nivel de lectura.

b) La intencionalidad original del lector podría ser el factor más importante para determinar niveles de lectura.

c) Las consideraciones estilísticas del autor de un texto no sería factor a la hora de determinar su dificultad.

Como referencia, los rangos ARI son los siguientes:

\begin{tabular}{|c|c|c|c|c|c|c|c|c|c|c|c|c|c|c|}
\hline $\begin{array}{l}\text { Sco } \\
\text { re }\end{array}$ & 1 & 2 & 3 & 4 & 5 & 6 & 7 & 8 & 9 & 10 & 11 & 12 & 13 & 14 \\
\hline Age & $5-6$ & $\begin{array}{l}6- \\
7\end{array}$ & $\begin{array}{l}7- \\
8\end{array}$ & $\begin{array}{l}8- \\
9\end{array}$ & $\begin{array}{l}- \\
10\end{array}$ & $\begin{array}{l}10 \\
- \\
11\end{array}$ & $\begin{array}{l}11 \\
- \\
12\end{array}$ & $\begin{array}{l}12 \\
- \\
13\end{array}$ & $\begin{array}{l}13 \\
- \\
14\end{array}$ & $\begin{array}{l}14 \\
- \\
15\end{array}$ & $\begin{array}{l}15- \\
16\end{array}$ & $\begin{array}{l}16- \\
17\end{array}$ & $\begin{array}{l}17- \\
18\end{array}$ & $18-22$ \\
\hline $\begin{array}{l}\text { Gr } \\
\text { Lev } \\
\text { el }\end{array}$ & $\begin{array}{l}\text { Kind } \\
\text { er }\end{array}$ & $\begin{array}{l}1 \mathrm{~s} \\
\mathrm{t} \\
\mathrm{gr}\end{array}$ & $\begin{array}{l}2 \mathrm{n} \\
\mathrm{d} \\
\mathrm{gr}\end{array}$ & $\begin{array}{l}3 \mathrm{r} \\
\mathrm{d} \\
\mathrm{gr}\end{array}$ & $\begin{array}{l}4 \mathrm{t} \\
\mathrm{h} \\
\mathrm{gr}\end{array}$ & $\begin{array}{l}5 \mathrm{t} \\
\mathrm{h} \\
\mathrm{gr}\end{array}$ & $\begin{array}{l}\text { 6t } \\
\mathrm{h} \\
\mathrm{gr}\end{array}$ & $\begin{array}{l}7 \mathrm{t} \\
\mathrm{h} \\
\mathrm{gr}\end{array}$ & $\begin{array}{l}8 \mathrm{t} \\
\mathrm{h} \\
\mathrm{gr}\end{array}$ & $\begin{array}{l}9 \mathrm{t} \\
\mathrm{h} \\
\mathrm{gr}\end{array}$ & $\begin{array}{l}10 t \\
h \\
g r\end{array}$ & $\begin{array}{l}11 \mathrm{t} \\
\mathrm{h} \\
\mathrm{gr}\end{array}$ & $\begin{array}{l}12 \mathrm{t} \\
\mathrm{h} \\
\mathrm{gr}\end{array}$ & $\begin{array}{l}\text { Colleg } \\
\text { ue }\end{array}$ \\
\hline
\end{tabular}

Cuadro 2: Referencia niveles ARI

Para poder otorgar el rango apropiado para los textos, y condensar la información sobre terminología y legibilidad, se condensó toda la información para la construcción de un instrumento en forma de escala de apreciación, debido a las características de uso y fácil selección, sesgando al mínimo el juicio experto de un académico para la selección de textos. En dicha construcción, se otorgaron rangos basados en ARI para la consideración de la complejidad estableciendo lo siguiente:

\begin{tabular}{|c|c|}
\hline $12-14$ & Texto complejo \\
\hline $9-11$ & Complejidad intermedia \\
\hline Bajo 9 & Baja complejidad \\
\hline
\end{tabular}

Estos rangos están directamente relacionados con los niveles de inglés expuestos anteriormente, y según el investigador y su experiencia como académico, el aprendizaje del inglés como segunda lengua sería un factor fundamental a la hora de que el alumnado comprenda los textos, por lo que sus capacidades de comprender, rastrear y leer afectarán directamente la adquisición 
Complejidad textual con fines académicos en el ámbito de la traducción...

de las competencias establecidas para el educando. Entonces, considerando un proceso de selección de textos, se definió un instrumento en forma de escala de apreciación, dada la accesibilidad y uso de la misma. Se definieron criterios, indicadores, escala de valoración, ponderación y puntaje. Será de especial énfasis el puntaje ya que se podrán definir nuevas categorías y así también nuevas escalas a partir de definiciones más técnicas.

\begin{tabular}{|c|c|c|c|c|c|}
\hline & & Siempre & $\begin{array}{l}\text { General- } \\
\text { mente }\end{array}$ & $\begin{array}{l}\text { Ocasional- } \\
\text { mente }\end{array}$ & Nunca \\
\hline $\begin{array}{l}\text { Densidad } \\
\text { terminológica }\end{array}$ & $\begin{array}{l}\text { La cantidad de } \\
\text { términos } \\
\text { específicos } \\
\text { identificados } \\
\text { supera el } \\
\text { umbral de } 4 \\
\text { términos cada } \\
10 \text { palabras. }\end{array}$ & & & & \\
\hline $\begin{array}{l}\text { Nivel de } \\
\text { especialización }\end{array}$ & $\begin{array}{l}\text { La } \\
\text { terminología } \\
\text { específica } \\
\text { requiere del } \\
\text { uso de fuentes } \\
\text { especializadas } \\
\text { o de uso en } \\
\text { contexto para } \\
\text { la obtención } \\
\text { del } \\
\text { significado. }\end{array}$ & & & & \\
\hline & $\begin{array}{l}\text { La cantidad de } \\
\text { remisiones de } \\
\text { la } \\
\text { terminología } \\
\text { del texto es } \\
\text { limitada dado } \\
\text { su uso } \\
\text { específico. }\end{array}$ & & & & \\
\hline $\begin{array}{l}\text { Legibilidad } \\
\text { según índices } \\
\text { automáticos }\end{array}$ & $\begin{array}{l}\text { El resultado } \\
\text { ARI supera el } \\
\text { valor } 16\end{array}$ & & & & \\
\hline
\end{tabular}

Cuadro 3: Escala de apreciación de selección de textos según complejidad 
FERNANDO MUÑOZ LÓPEZ

Se incluyó un criterio de fuentes de información, que resultará ser fundamental para la determinación de la complejidad, ya que, por medio de esta escala, cada usuario puede discernir por sí solo la fiabilidad de la fuente de información. Ya siendo usuario de un texto en extremo complejo, el proceder profesional de inmediato nos lleva a buscar en cierto tipo de fuentes de especialidad, usualmente primarias. La problemática que intentamos también subsanar es que para el usuario de la escala o traductor profesional es un proceder normal, pero el estudiante de traducción es un principiante en uso de fuentes. Por lo tanto, si se asume que nuestros estudiantes poseen nuestro mismo conocimiento, nos encontraremos con traducciones de alumnos de pregrado que no cumplen con requerimientos mínimos de calidad cumplan las expectativas. Esto no necesariamente es por la poca prolijidad del alumno, sino puede ser perfectamente porque seleccionamos un texto que no es acorde a su nivel de adquisición de la lengua extranjera, o que sus fuentes de información deben ser revisadas por el profesor guía y no asumir conocimiento sobre fuentes de información.

\subsection{Muestras, resultados, análisis}

La muestra para este proyecto son los programas de estudio de la Universidad Tecnológica de Chile, INACAP, (2017), en su carrera de pregrado Traducción Inglés-Español, mención Negocios Internacionales. Se seleccionarán dos programas de estudio, del total de siete, que incluyan distintos niveles de complejidad, para poder realizar el trabajo. Los programas son los siguientes:

Taller de Traducción III. Indicador de desarrollo traducción: Traduce al español textos de mediana complejidad y extensión sobre temas técnico-científicos, conforme a las normas de traducción, mediante el uso de herramientas tecnológicas.

Taller de Traducción IV. Indicador de desarrollo traducción: Traduce al español textos extensos y complejos, sobre temas técnico-científicos, conforme a las normas de traducción y al correcto uso del español, mediante el uso de herramientas tecnológicas.

Estos talleres de traducción se enmarcan en el segundo y tercer año de carrera, y se seleccionaron ya que ambos incluyen en sus indicadores la complejidad (solamente indicada, no explicada) y también tienen un nivel de adquisición de la lengua inglesa en distintos niveles, por lo que fue ideal para testear la escala de complejidad con distintos niveles de textos. Los participantes de la aplicación fueron seleccionados para otorgar mayor fiabilidad al instrumento, al no ser aplicado por el investigador que suscribe. La selección de participantes se limita 
Complejidad textual con fines académicos en el ámbito de la traducción...

a expertos en el área de aprendizaje del inglés y la traducción en la Universidad INACAP, sede La Serena, y también expertos en la creación de instrumentos para el aprendizaje de la traducción basado en competencias, que es el modelo que sigue la Universidad INACAP. A continuación, se presentan los resultados obtenidos de la aplicación del investigador y se verifica que los resultados obtenidos de esta investigación sean similares en profesionales que tributan en cuanto a competencias académicas en los programas de talleres de traducción. A continuación, se presenta el detalle de los participantes:

a) Sujeto 2: Profesor de Estado en inglés, Master in Arts in Applied Linguistics.

b) Sujeto 3: Profesor de Estado en inglés, Licenciado en Educación. Universidad INACAP, sede La Serena.

c) Sujeto 4: Traductora inglés-español. Magíster en Educación Superior. INACAP.

d) Sujeto 5: Traductora inglés-español INACAP.

e) Sujeto 6: Traductor inglés-español, Licenciado en Lengua Inglesa. Máster en Tecnologías de la Educación y TIC.

La aplicación del instrumento se realizó a 4 tipos distintos de textos del área de las humanidades y el área científica. La muestra de textos se seleccionó de acuerdo con el perfil de egreso de los alumnos de INACAP Chile, entendiendo su formación técnico-científica y rasgos humanistas en asignaturas transversales. Cabe destacar que hay 2 profesores de inglés incluidos en la muestra, debido a que su participación en la formación traductora resulta fundamental para desarrollar habilidades lingüísticas de producción o recepción de la lengua extranjera. A continuación, se incluyen los resultados de la aplicación por parte de los participantes con su puntaje y cuatro abstract de muestra de los distintos tipos de textos como referencia para visualizar las características sintácticas y terminológicas de los mismos. Se decidió considerar solo los fragmentos iniciales de cada artículo, considerando que poseen la información resumida y contenida en todo el artículo, por utilización de conceptos clave y contenido.

\begin{tabular}{|l|l|l|l|l|}
\hline & $\begin{array}{l}\text { Texto } \\
\text { "Artificial } \\
\text { Light" }\end{array}$ & $\begin{array}{l}\text { Texto "Null } \\
\text { mutation" }\end{array}$ & $\begin{array}{l}\text { Texto } \\
\text { "Catharsis" }\end{array}$ & $\begin{array}{l}\text { Texto } \\
\text { "Trauma" }\end{array}$ \\
\hline Estándar 1 & $\mathbf{9}$ & $\mathbf{1 3}$ & $\mathbf{7}$ & $\mathbf{1 2}$ \\
\hline Sujeto 1 & 5 & 11 & 6 & 5 \\
\hline Sujeto 3 & 6 & 12 & 5 & 5 \\
\hline Sujeto 4 & 8 & 12 & 6 & 9 \\
\hline Sujeto 5 & 9 & 13 & 8 & 9 \\
\hline
\end{tabular}


FERNANDO MUÑOZ LÓPEZ

\begin{tabular}{|l|l|l|l|l|}
\hline Sujeto 6 & 7 & 10 & 8 & 9 \\
\hline \multicolumn{4}{|c|}{ Tabla 1: Resultados generales aplicación escala de complejidad } \\
\hline
\end{tabular}

a) Texto Artificial Light

A central aim of the "lighting revolution" (the transition to solid-state lighting technology) is decreased energy consumption. This could be undermined by a rebound effect of increased use in response to lowered cost of light. We use the first-ever calibrated satellite radiometer designed for night lights to show that from 2012 to 2016, Earth's artificially lit outdoor area grew by $2.2 \%$ per year, with a total radiance growth of $1.8 \%$ per year.

Continuously lit areas brightened at a rate of $2.2 \%$ per year. Large differences in national growth rates were observed, with lighting remaining stable or decreasing in only a few countries. These data are not consistent with global scale energy reductions but rather indicate increased light pollution, with corresponding negative consequences for flora, fauna, and buman well-being (Kyba et al 2017).

\section{b) Texto Null Mutation}

Plasminogen activator inbibitor-1 (PAI-1) has been shown to be a key component of the senescence-related secretome and a direct mediator of cellular senescence. In murine models of accelerated aging, genetic deficiency and targeted inbibition of PAI-1 protect against aging-like pathology and prolong life span. However, the role of PAI-1 in human longevity remains unclear. We bypothesized that a rare loss-off-function mutation in SERPINE1 (c.699_700dupTA), which encodes PAI-1, could play a role in longevity and metabolism in bumans. We studied 177 members of the Berne Amish community, which included 43 carriers of the null SERPINE1 mutation. Heterosygosity was associated with significantly longer lenkocyte telomere length, lower fasting insulin levels, and lower prevalence of diabetes mellitus. In the extended Amish kindred, carriers of the null SERPINE1 allele had a longer life span. Our study indicates a causal effect of PAI-1 on buman longevity, which may be mediated by alterations in metabolism. Our findings demonstrate the utility of studying loss-of-function mutations in populations with geographic and genetic isolation and shed light on a novel therapeutic target for aging (Khan et al 2017).

c) Texto Catharsis

In residential child care, physical restraint continues to be a contentious and bigh-risk intervention with potential for physical and psychological harm to all involved. Its relationship 
Complejidad textual con fines académicos en el ámbito de la traducción...

to catharsis is poorly understood and rarely addressed in policy, practice and literature. Indeed, there is a paucity of application of catharsis theory to residential child care (or social work generally). This article redresses this gap by presenting findings of a large-scale, qualitative study of children, young people and practitioners' experiences of physical restraint and analysing them through lenses of catharsis and containment theories. It offers evidence of cathartic expression in situations involving restraint, as well as a potential relationship between ongoing, repeated restraints and a drive for catharsis. It is argued that catharsis theory, especially when combined with containment theory, has explanatory power in making sense of physical restraint and how to minimise its use while still meeting the needs of children and direct-care practitioners in residential and other relevant settings (Steckley et al 2018).

\section{d) Texto Trauma}

Social workers frequently encounter clients with a history of trauma. Trauma-informed care is a way of providing services by which social workers recognize the prevalence of early adversity in the lives of clients, view presenting problems as symptoms of maladaptive coping, and understand how early trauma shapes a client's fundamental beliefs about the world and affects his or her psychosocial functioning across the life span. Trauma-informed social work incorporates core principles of safety, trust, collaboration, choice, and empowerment and delivers services in a manner that avoids inadvertently repeating unbealthy interpersonal dynamics in the belping relationship. Trauma-informed social work can be integrated into all sorts of existing models of evidence-based services across populations and agency settings, can strengthen the therapeutic alliance, and facilitates posttraumatic growth (Levenson 2017).

Los resultados generales de la aplicación del material instruccional indican en algunos aspectos claras diferencias en cuanto al juicio experto de los sujetos evaluadores. Se estableció un estándar a partir de la aplicación del propio investigador; se establece un mínimo de error estándar en análisis de datos correspondiente al $15 \%$ del resultado real (para esto se aproxima a 2 puntos diferenciales por categoría). Como se puede apreciar, en pocos campos se sobrepasa el mínimo de error, usualmente en profesionales de la pedagogía, entendiendo quizás que muchas veces el resultado se puede ver sesgado por el propósito profesional de cada persona, en este caso, quizás no es su primera opción considerar el texto para "traducir". Para justificar el uso de la tabla, se procedió a analizar la cantidad de terminología en cada oración según lo determinado anteriormente por el investigador. Luego cada término fue buscado y se determinó la fuente de información apropiada para el texto y su posterior traducción, contabilizando también sus resultados ARI y el porcentaje de terminología que posee el texto. Este resultado estándar se asignó a cada tabla para 
tener un punto de comparación entre el proceso realizado por el investigador y el proceso usual de selección de textos de los sujetos en el estudio. A continuación, se presenta el detalle del análisis de un texto como ejemplo, "Trauma", ya que posee la mayor diferencia de puntaje con respecto al estándar.

Resultados ARI: 23,67

Total de palabras: 320

Total de términos: 42

Porcentaje especialización texto: $26,56 \%$

Los resultados del índice de legibilidad son claramente para textos complejos, ya que la sintaxis del documento para su traducción involucra varios retos para el traductor (párrafo 4 en muestra referencial de textos). Como se puede apreciar en dicho párrafo, la estructura del inglés está claramente marcada por la pasividad, unidades terminológicas compuestas, y de fuentes de información técnicas y de contexto: de las fuentes utilizadas, lo siguiente corresponde a las fuentes para su correspondiente traducción equivalente:

Fuente genérica: 10 términos

Fuente especializada: 32 términos

Del total de 42 términos, solo de 10 fueron encontrados sus equivalentes funcionales en fuentes de información generales, mientras que de 32 de los mismos se necesita desarrollo documental más exhaustivo para las equivalencias. Se entiende que de los textos de esta índole pueden no necesariamente encontrarse remisiones exactas, y se debiese incurrir a la habilidad discursiva del traductor, por lo que la dificultad estilística sería mucho mayor al traducir este tipo de textos que los científicos técnicos. Los resultados por ende difieren de este análisis, entendiendo el investigador que dichos resultados se deben principalmente al análisis de "lectura" que pueden hacer los sujetos que aplicaron la escala. Cada sujeto se encuentra en el nivel C1 del Marco Común de Referencia Europeo, con certificación, por lo que el investigador sostiene que el juicio experto en esta selección de complejidad jugó un rol fundamental afectando los resultados del estándar. A continuación, se incluye un ejemplo contrario, donde el puntaje asociado es homogéneo del texto Null Mutation en todas las muestras, siguiendo el mismo patrón de análisis: 
Complejidad textual con fines académicos en el ámbito de la traducción...

\begin{tabular}{|l|l|l|l|l|l|l|}
\hline & $\begin{array}{l}\text { Densidad } \\
\text { terminoló- } \\
\text { gica }\end{array}$ & $\begin{array}{l}\text { Nivel de } \\
\text { especializa- } \\
\text { ción }\end{array}$ & & Legibilidad & Resultado \\
\hline Estándar 1 & $\mathbf{3}$ & $\mathbf{4}$ & $\mathbf{4}$ & $\mathbf{4}$ & $\mathbf{1}$ & $\mathbf{1 3}$ \\
\hline Sujeto 2 & 4 & 3 & 4 & 1 & 4 & 11 \\
\hline Sujeto 3 & 3 & 4 & 3 & 4 & 1 & 12 \\
\hline Sujeto 4 & 4 & 4 & 4 & 4 & 1 & 13 \\
\hline Sujeto 5 & 3 & 4 & 3 & 1 & 4 & 10 \\
\hline Sujeto 6 & 4 & 4 & 4 & 1 & 4 & 12 \\
\hline
\end{tabular}

Tabla 2: resultados texto Null Mutation

En general, se puede inferir que, independientemente de la entrega de un material instruccional, la variable del juicio experto juega un rol fundamental a la hora de categorizar un texto con las dimensiones antes expuestas. El grado de complejidad establecido en esta investigación está determinado principalmente por la terminología, la competencia documental atribuida a esta, el conocimiento estructural del inglés escrito y una herramienta de libre acceso para determinar la legibilidad. Se sostiene que para verificar el $100 \%$ de efectividad de la escala, una muestra de distintos tipos de textos correspondientes a distintas tipologías textuales debiesen analizarse, y también una muestra mayor de traductores y pedagogos, por separado, debiesen realizar nuevamente la aplicación, aunque en términos generales, la escala resultó ineficiente en textos como "Trauma", el cual está inserto en el ámbito de las humanidades, donde la característica estilística del autor representa un reto al traductor, no tanto así al lector. El investigador por ende sostiene que para textos de características estilísticas particulares ligadas al ámbito del pensamiento humanista debiese agregar alguna otra dimensión en la escala que se enfoque en este punto, llámese registro o uso del lenguaje.

\section{Conclusión}

La articulación de la complejidad y la selección buscaba como objetivo principal definir los criterios de clasificación de textos y variables incidentes para el proceso académico traductor. De acuerdo con lo investigado, los criterios de densidad terminológica, nivel de especialización e índice de legibilidad otorgan un punto de partida valioso a la hora de operacionalizar el proceso de selección de textos para los programas de estudio de la carrera de traducción. Esto es así porque delimitan los ámbitos de comprensión del texto, permitiendo realizar un ajuste para la lectura comprensiva del material, que incide directamente en el resultado de un producto de traducción. La selección de textos por parte del profesorado no tiene necesariamente un estándar sistematizado, por lo que lo 
anterior queda a juicio experto de cada uno, incidiendo directamente en tareas específicas de traducción. Considerando que cada programa tiene una competencia asociada, un perfil de egreso establecido y declarado por cualquier institución educacional que imparta traducción en sus programas, y a su vez un perfil docente, se hace necesaria una estandarización de criterios para el aprendizaje del inglés para el proceso de traspaso de una L1 a L2, para así alcanzar las competencias declaradas por cada programa. En el presente trabajo, se puede inferir que existe un grado de homogeneidad de resultados con un margen de error no mayor a dos puntos en 3 de los 4 textos, pero sí una mayor diferencia en el texto que posee convenciones y estilo humanista-social, con mayor libertad discursiva. Para entender el fenómeno de diferencias de puntajes en los distintos tipos de texto, se considera como fundamental el conocimiento y comprensión previa de los sujetos y su efecto en la clasificación de los mismos. Los procesos cognitivos responsables de la comprensión también resultan clave a la hora de clasificar textos, ya que se entiende que la propia experiencia teórica proporciona la información elemental para su comprensión y la misma aplicación en el aula (Elena 2011). Esto podría evidenciar que prima la experiencia propia para juzgar el propósito del texto, sin considerar las necesidades reales del alumnado asociado a una habilidad traductora específica. Según el investigador, los resultados de categorización pueden ser resultado de la noción de conocimiento sobre el lenguaje de especialidad y el lenguaje común, que en apariencia son similares pero cada uno con distintos grados de profundidad (Alcina y Gamero 2002: 73). Para dicha aplicación, el investigador infiere que la escala de apreciación debe incluir un apartado de estilo a desarrollar. Uno de los principales objetivos del trabajo es poder simplificar la labor del profesor a la hora de la selección, para que el trabajo aplicado en clase sea más adecuado y con parámetros establecidos, y así poder alcanzar las competencias declaradas por los programas académicos y objetivos dentro de una clase tipo taller de traducción. La selección de textos siempre estará dentro de los elementos de juicio experto que todos los traductores tienen por formación. El punto crítico de este escrito permite proyectar cuánto puede incidir en la profesionalización de un estudiante, un procedimiento de adecuación de material textual en adquisición de una lengua junto con el desarrollo de la competencia procedimental de traducción. Dicha articulación por supuesto dependerá de la dificultad de la lengua, o las competencias de entrada que posean los futuros profesionales de la traducción.

\section{Referencias bibliográficas}


Complejidad textual con fines académicos en el ámbito de la traducción...

AlCINA, A.; GAMERO, S., La traducción cientifico-técnica y la terminología en la sociedad de la información. Castelló de la Plana: Publicacions de la Universitat Jaume I, 2002.

ARI AUTOMATED READABILITY INDEX, 1967. Disponible en línea en: https://www.webpagefx.com/tools/read-able/automated-readabilityindex.html

Cabré Castellvi, M. T., Terminology, theory, methods and applications. Barcelona: John Benjamins Publishing Company, 1992.

CABRERÓ J.; LLORENTE M., La aplicación del juicio experto como técnica de evaluación de las tecnologías de la información y comunicación. Sevilla: Universidad de Sevilla, 2013.

Council of Europe. Common European Framework of Reference. Europe C.O., 2018.

ELENA, P., "Bases para la comprensión organizativa del texto". En: Revista de Lingüistica y Lenguas Aplicadas, 6, 2011. Disponible en línea en: https://polipapers.upv.es/index.php/rdlyla/article/view/898/968

GONZALO GARCIA, C., Manual de documentación y terminología para la traducción especializada. Madrid: Arco/Libros, 2004.

INACAP, Programa de estudio Traducción Inglés-Español, mención Negocios Internacionales. Chile. Universidad Tecnológica de Chile, INACAP, 2017.

IZQUIERDO GARCÍA, I., Competencia textual para la traducción. Valencia: Tirant Lo Blanch, 2011.

KHAN, S.; SHAH, S.; KLYYACHKO, E. et al., "A null mutation in SERPINE1 protects against biological aging in humans”. En: Sciences Advances, 3, 11, 2017. Disponible en línea en:

https:/ /advances.sciencemag.org/content/3/11/eaao1617

KYBA, C.; KuESTER; T., SÁNCHEZ, A. et al., "Artificially lit surface of Earth at night increasing in radiance and extent”. En: Sciences Advances, 3, 8, 2017. Disponible en línea en: https://advances.sciencemag.org/content/3/11/e1701528.

LEVEnSON, J., "Trauma-Informed Social Work Practice". En: Social Work, 62, 2 , 2017, pp. 105-113. Disponible en línea en:

https:/ /academic.oup.com/sw/article/62/2/105/2937786

MAYORAL ASENSIO, R., Experiencias de traducción. Reflexiones desde la práctica traductora. Castelló de la Plana: Publicacions de la Universitat Jaume I, D.L., 2005.

MiniSTERIO DE EdUCACIÓN, C. Y., Marco común europeo de referencia para las lenguas: aprendizaje, enseñanza, evaluación. Madrid: Secretaría General Técnica del MECD y Grupo ANAYA S.A., 2002. 
FERNANDO MUÑOZ LÓPEZ

STECKLEY, L., "Containment and Physical Restraint in Residential Child Care". En: The British Journal of Social Work, 6, 48, 2018, pp. 1645-1663. Disponible en línea en: https:// academic.oup.com/bjsw/article/48/6/1645/4657142

VERMEER, H., "Reflexiones preliminares sobre retórica y estilística en la traducción”. En: Acta Poética, 25, 1, 2004, pp. 15-44. Disponible en línea en: http://www.scielo.org.mx/scielo.php?script=sci_arttext\&pid=S018530822004000100001

WARING, R., "A study of receptive and productive learning from word cards". En: Studies in Foreign Languages and Literature, 21, 1997, pp. 94-114.

YULE, G., El lenguaje. España: AKAL AC, 2007. 